Review

\title{
Krüppel-like Factor 15: A Potential Therapeutic Target For Kidney Disease
}

\author{
Lefeng Wang1, Weiqiang $\operatorname{Lin}^{1,2}, \bowtie$, Jianghua Chen ${ }^{1,} \bowtie$ \\ 1. The Kidney Disease Center, The First Affiliated Hospital, Zhejiang University School of Medicine, Hangzhou 310003, China. \\ 2. Institute of Translational Medicine, Zhejiang University School of Medicine, Hangzhou 310029, China.
}

$\triangle$ Corresponding authors: Dr. Weiqiang Lin, The Kidney Disease Center, the First Affiliated Hospital, Institute of Translational Medicine, Zhejiang University School of Medicine, Hangzhou 310029, China. Tel: +1 86571 86971990; Email: wlin@zju.edu.cn. Dr. Jianghua Chen, The Kidney Disease Center, the First Affiliated Hospital, Zhejiang University School of Medicine, Hangzhou 310003, China. Tel: +86 571 86971990; Email: chenjianghua@zju.edu.cn.

(C) The author(s). This is an open access article distributed under the terms of the Creative Commons Attribution License (https://creativecommons.org/licenses/by/4.0/). See http://ivyspring.com/terms for full terms and conditions.

Received: 2019.03.12; Accepted: 2019.06.28; Published: 2019.07.21

\begin{abstract}
Krüppel-like factor 15 (KLF15) is a zinc-finger transcription factor highly expressed in the glomeruli and interstitial cells of kidneys. An increasing number of studies have demonstrated a critical role for KLF15 in the kidney, involving tubular physiology, podocyte injury, renal fibrosis, and mesangial pathology. In this review, we discuss recent advances and update our overview of the functions of KLF15 in kidney biology, hoping to provide new perspectives on the progression and therapy of Chronic Kidney Disease (CKD). A better understanding of KLF15 with respect to its diverse roles in specific cells or diseases will be beneficial in pursuing novel therapeutic targets and moving forward to precision medicine.
\end{abstract}

Key words: Krüppel-like factor 15; Kidney disease; Podocyte differentiation; Mesangial cell; Renal fibrosis

\section{Introduction}

Chronic Kidney Disease (CKD) is highly prevalent in many countries (1-4), increasing the risk of cardiovascular disease, cognitive dysfunction, as well as general mortality $(4,5)$. Individuals with CKD may gradually develop end-stage renal disease (ESRD) and need to receive renal replacement therapy (RRT) $(3,6,7)$. RRT is available in developed countries but difficult to access in poor areas $(8,9)$, and greatly affects the quality of life of patients. Therefore, CKD has become a global public health problem, and efficient therapeutic targets are urgently needed to slow CKD progression and improve prognoses (10, $11)$.

Krüppel-like factors (KLFs) are a group of zinc-finger DNA-binding transcription factors involved in various biological processes, such as cell differentiation, metabolism, inflammation, apoptosis, mitochondrial biogenesis, DNA repair, and many others (12-15). The KLFs family consist of eighteen members, among which KLF15, is widely distributed in the glomeruli and interstitium of kidneys, pancreas, heart, liver, and skeletal muscles (16). Previous studies have demonstrated that KLF15 is a key transcriptional regulator in diverse physiological processes, including gluconeogenesis, immune response and adipocyte differentiation (17-20). Moreover, KLF15 was also involved in metabolic dysfunctions, including diabetes, inflammation, obesity, and cardiac hypertrophy (17-20). Recently, a growing number of studies have indicated that KLF15 is implicated in renal physiological processes and pathologic progression of CKD, involving podocyte differentiation, tubular physiology, mesangial pathology, and renal fibrosis (12, 13, 16, 21). An improved understanding of CKD and its molecular mechanisms will enable further research into new therapeutic targets.

Herein, we summarize relevant studies and highlight recent updates on KLF15 and its role in renal biology, hoping to provide insights into potential areas for further investigation.

\section{Structure and function of KLF15}

Human KLF15 is mapped at 3q21.3 according to human chromosome mapping (22). The KLF15 cDNA is composed of a single open reading frame of 1248 
$\mathrm{bp}$, encoding a polypeptide of 416 amino acids (22, 23). Like other members of the KLFs family, KLF15 protein contains a conserved three-zinc-finger $(\mathrm{C} 2 \mathrm{H} 2)$ motif in the C-terminal region, which can bind to GC-rich sequences in target gene promoters and regulate transcription levels (24). Though the DNA-binding ability is similar, KLFs family have distinct transactivation or transrepression domains in their N-terminal region, leading to different tissue distribution patterns and biological functions of KLFs. According to the differences, KLFs can be divided into three groups (25). Members of the first group act as transcriptional repressors by interacting with C-terminal binding protein (CtBP) (25). KLFs in the second group work as transcriptional activators (25), and members of the third group serve as transcriptional repressors through an a-helical motif, which can mediate their binding to Sin3A (25). However, KLF15 is not a member of any of these three groups, because the protein interaction domains of KLF15 have not been determined yet. Thus, a more specific illustration of its structure requires further research.

\section{Roles of KLF15 in kidney physiology}

\section{KLF15 in renal tubular physiology}

KLF15 was first described as Kidney-enriched Krüppel-like factor (KKLF), a gene found to be expressed in human mesangial cells, interstitium, and the nephron segment where the kidney-specific chloride channels CLC-K1 and CLC-K2 were absent (22). In rat kidney cells, KLF15 appeared to suppress the expression of CLC-K1 and CLC-K2 in the thin descending limb of Henle's loop and inner medullary collecting ducts by competing for promoter binding with myc-associated zinc-finger (MAZ) protein, a transcription factor able to activate CLC-K1 and CLC-K2 $(22,23)$. This finding indicates that KLF15 is associated with the nephron segment-specific expression of CLC-K1 and CLC-K2 genes (23). However, it still remains unclear whether KLF15 is functional in chloride transport (12).

\section{KLF15 in podocyte differentiation}

Podocyte is highly differentiated in normal mature kidneys (12). The loss of podocyte differentiation markers is regarded as a main manifestation of podocyte injury (26). Previous studies have shown that retinoic acid (RA) may induce podocyte differentiation via activation of the cAMP pathway, however, there appear to be other pathways, as binding sites for cAMP-response elements (CREB) are lacking in promoters of many podocyte maturity marker genes (27). One hypothesis is that podocyte differentiation may be mediated by
RA-induced transcription factors, and microarray gene expression studies in human podocytes identified a differentially regulated gene, KLF15, which was up-regulated in both murine and human podocytes treated with RA $(27,28)$. Subsequently, KLF15 was shown to regulate RA-induced restoration of podocyte maturity markers by transcriptionally activating podocyte-specific genes (Synaptopodin, Podocin, and Nephrin) under cell stress (27) (Fig. 1).

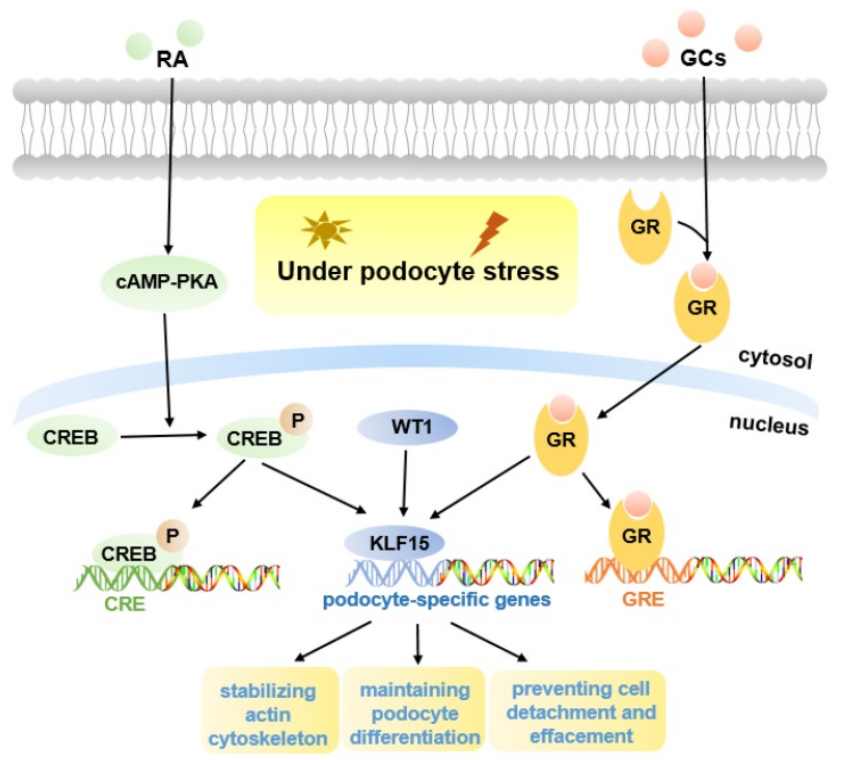

Figure 1. Molecular mechanisms of KLF15 in podocyte injury. KLF15 mediates RA and GCs-induced restoration of podocyte differentiation markers (Nephrin, Synaptopodin, and Podocin) under podocyte stress. Pathways activated by KLF15 include stabilization of the actin cytoskeleton, podocyte differentiation, and focal adhesion. The activity of KLF15 is partly mediated by WT1.

\section{The functions of KLF15 in Drosophila nephrocytes}

Interestingly, a study demonstrated a vital role of KLF15 in the development and differentiation of the Drosophila nephrocyte, a research model of human podocytes (29). The nephrocyte of Drosophila is a highly endocytic cell, which expresses genes that are evolutionarily conserved in human podocytes (30). In the Klf15 loss-of-function mutants, nephrocyte development was impaired during embryogenesis. In addition, a loss of endocytic scavenger function was also observed in the adult Drosophila nephrocytes when Klf15 was conditionally silenced (29). Inversely, overexpression of Klf15 led to the proliferation of nephrocytes and the improvement of cell function during ageing process (29). Additionally, Klf15 was involved in a nephrocyte-restricted differentiation pathway, indicating that Klf15 was essential in maintaining the differentiation of Drosophila nephrocytes (29). Future research may use Drosophila to explore further functions and mechanisms of 
KLF15 in human podocytes.

\section{Roles of KLF15 in kidney disease}

\section{KLF15 in podocyte injury}

Pathological mechanisms of CKD include cellular injury, inflammation, podocyte effacement, proteinuria, and glomerulosclerosis (31). Podocyte injury is characterized by loss of mature podocyte differentiation markers and destabilization of the actin cytoskeleton in response to excessive stress. It is regarded as the hallmark of various glomerular diseases, such as Focal Segmental Glomerulosclerosis (FSGS), Membranous Nephropathy (MN), Minimal Change Disease (MCD), and HIV-Associated Nephropathy (HIVAN) $(27,32,33)$.

Previous studies indicated that podocyte-specific Klf15-KO mice in the unperturbed state did not develop significant proteinuria or glomerulosclerosis, but in Lipopolysaccharide (LPS) or Adriamycin (ADR)-induced proteinuric murine models, loss of KLF15 led to a significant increase in the susceptibility to podocyte injury, indicating a critical role for KLF15 as a regulator of podocyte differentiation protecting against podocyte injury (27) (Fig. 1). Similarly, a lower expression of KLF15 in podocytes from patients with primary glomerulopathies, including FSGS and HIVAN, also confirmed this view (27); a lower expression seems to be correlated with worse renal outcomes for patients (34).

Glucocorticoids (GCs) are usually the primary therapy for glomerular diseases (35). Recent studies demonstrated that, in both murine and human podocytes treated with GCs, the KLF15 levels were dramatically increased, and the affinity of KLF15 promoters binding with GCs-receptor was also improved (16). Loss of KLF15 in murine podocytes weakened the beneficial effect of GCs, resulting in destabilization of the actin cytoskeleton and increased podocyte injury (16). In contrast, overexpression of KLF15 under podocyte stress led to improved stabilization of the actin cytoskeleton (16). Moreover, KLF15 levels in the glomeruli of patients with glomerular diseases were associated with the responsiveness to GCs (16). Consequently, these studies identified important effects of KLF15 in mediating the benefits of GCs in podocytes (Fig. 1).

Excitingly, a recent report showed that podocyte-specific induction of Klf15 in ADR-induced and HIV-1 transgenic (Tg26) proteinuric murine models, can attenuate renal fibrosis, inflammation and podocyte injury, contributing to improved cell survival (13). Enrichment analyses have indicated that induction of Klf15 is implicated in the restoration of podocyte differentiation markers, stabilization of the actin cytoskeleton, as well as focal adhesion. Therefore, KLF15 may be a novel therapeutic target in proteinuric diseases (13). Moreover, Wilms Tumor 1 (WT1) was observed to regulate the activity of KLF15 in part (Fig. 1). WT1 is a critical podocyte-specific transcription factor, and plays an essential role in kidney development, indicating the potential functions of KLF15 in kidney development (13).

\section{KLF15 in mesangial pathology}

High glucose level is a critical contributor to diabetic nephropathy; in vitro evidence indicates that it can promote the proliferation of mesangial cells (36). KLF15 can inhibit the high glucose-induced cell proliferation, and repress the development of diabetic nephropathy (36). Possible mechanisms are associated with the extracellular-regulated kinase (ERK)/MAPK signaling pathways (36). Therefore, KLF15 may work in the therapy of diabetic nephropathy.

In a classic anti-Thy1 mesangial proliferative nephritis rat model, KLF15 expression in mesangial cells inhibited cell proliferation by down-regulating the expression of the cell cycle regulation factor E2F1, a downstream target of KLF15 (37) (Fig. 2). Thus, KLF15 is expected to be a potential therapeutic target for mesangial proliferative glomerulonephritis, a common non-specific change characterized by immune complex deposition, podocytopathies, and complement alterations (37).

\section{KLF15 in Lupus Nephritis}

Lupus nephritis (LN) is known to cause permanent renal injury. The issue of treatment-related toxicity in LN remains to be resolved, which has promoted the development of drug combinations (39-41). Previous studies have shown that KLF15 can block the interaction of nuclear factor NF-kB p56 with the co-activator P300 by directly interacting with P300, and subsequently by inhibiting the P300-p56-activated inflammatory pathway (42). The most recent evidence has indicated that KLF15 can improve LN (21). A combined treatment of Hydroxychloroquine and artemisinin in LN may exert renal-protective effects by increasing expression levels of KLF15, leading to a decrease in the levels of its downstream target, NF- $\kappa \mathrm{B}$, and consequently suppressing the inflammatory response (21).

\section{KLF15 in renal fibrosis}

Renal fibrosis, a dynamic process in response to excessive epithelial injury, consists of glomerulosclerosis and tubulointerstitial fibrosis due to extracellular matrix accumulation (43). Renal fibrosis is regarded as the histological manifestation and hallmark of progressive CKD, as well as a reliable 
indicator of prognosis $(7,44)$. Multiple factors are associated with the progression of fibrosis, such as oxidative stress, inflammation, and transforming growth factor- $\beta$ (TGF- $\beta$ ) (45). Herein, we provide an update on the research literature regarding the role of KLF15 on renal fibrosis (Fig. 2).
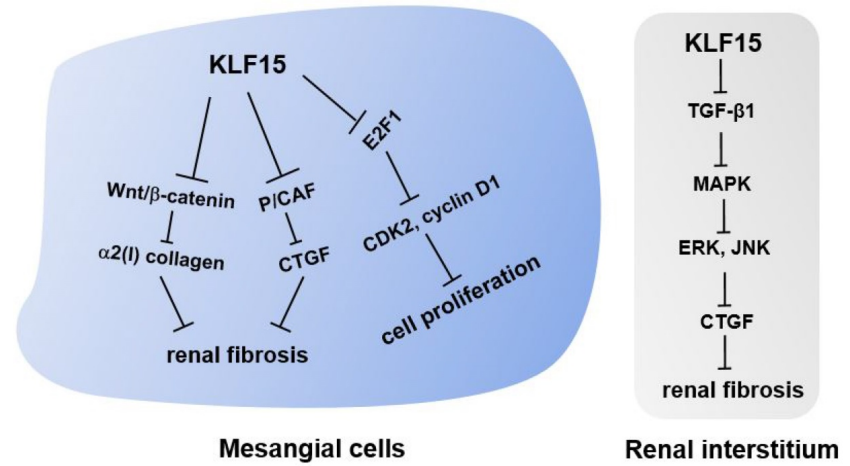

Figure 2. Molecular mechanisms of KLF15 in renal fibrosis and mesangial pathology. KLF15 plays an antifibrotic role in renal fibrosis through inhibiting the canonical $\mathrm{Wnt} / \beta$-catenin pathway and suppressing the recruitment of P/CAF to the CTGF promoter in mesangial cells. In the renal interstitium, KLF15 attenuates the deposition of extracellular matrix and CTGF by inhibiting TGF- $\beta 1$-mediated JNK/MAPK and ERK/MAPK signaling pathways. In addition, KLF15 expression in mesangial cells inhibits the proliferation of mesangial cells via repressing the cell cycle regulation factor E2F1.

When first described in the kidney, KLF15 was suggested to play a role in fibrogenesis, given its localization in potentially fibrogenic mesangial cells and tubulointerstitial compartments $(16,22)$. This notion was verified when authors observed a decreased expression level of KLF15 followed by increased levels of $\alpha 2(\mathrm{I})$ collagen in kidneys of unilateral ureteral obstruction (UUO) mice, a classic murine model of progressive renal fibrosis $(22,46)$. Subsequent experiments demonstrated that KLF15 repressed the activity of $\alpha 2(\mathrm{I})$ collagen promoter, thus inhibiting the synthesis of type I collagen $(22,23)$. Besides, knockdown of Klf15 in Foxd1 ${ }^{+}$stroma cells exacerbated the proliferation of myofibroblasts and the deposition of extracellular matrix in UUO and angiotensin II-treated mice, two murine models of renal fibrosis (46). Combined with in vitro experiments, these results confirmed that KLF15 was critical in attenuating renal fibrosis by inhibiting the canonical Wnt/ $\beta$-catenin pathway (46). In the angiotensin II-treated murine model, KLF15 was likely to inhibit the expression of angiotensin II-induced profibrotic connective tissue growth factor (CTGF) by suppressing the recruitment of the co-activator p300/CREB-binding protein-associated factor $(\mathrm{P} / \mathrm{CAF})$ to the CTGF promoter, thus exerting an early antifibrotic effect in renal fibrosis (18).

Interestingly, another report demonstrated that a low-protein diet increased renal KLF15 expression levels in normal and 5/6-nephrectomized rats, a remnant kidney model of progressive renal fibrosis (45). KLF15 levels in mesangial cells of remnant kidneys were suppressed due to elevated levels of TGF- $\beta$, tumor necrosis factor (TNF)- $\alpha$, and oxidative stress. Besides, overexpression of KLF15 down-regulated the levels of fibronectin as well as type IV collagen mRNAs in HEK293 and mesangial cells, and Klf15-KO mice were found to develop glomerulosclerosis after uninephrectomy $(45,47)$. These findings indicate that KLF15 may exert antifibrotic roles in mesangial cells. Other studies with similar results have also confirmed this hypothesis (36).

Apart from mesangial cells, the expression of KLF15 in the renal interstitium of 5/6-nephrectomized rats was also explored, and a significant decrease in KLF15 levels was observed (17). In vitro experiments demonstrated that KLF15 attenuated the deposition of extracellular matrix and CTGF by inhibiting TGF- $\beta 1$-mediated Jun N-terminal kinase (JNK)/mitogen-activated protein kinase (MAPK) and ERK/MAPK signaling pathways. Therefore, KLF15 may play an antifibrotic role in the interstitium of remnant kidneys through the molecular mechanisms mentioned above (17).

It is known that the main pathological characteristics of hypertension-induced kidney injury include glomerulosclerosis, tubulointerstitial fibrosis, and proteinuria (28). A recent study suggested that the deacetylation of KLF15 by Sirtuin 3 (SIRT3) may decrease the expression of fibronectin and type IV collagen in immortalized mouse podocyte MPC-5 cells and protect against hypertensive nephropathy (48).

\section{Challenges}

KLF15 was first identified in 2000 as a Kidney-enriched Krüppel-like factor, after which a great number of studies have focused on its physiological and pathological roles. Recent studies have greatly expanded our knowledge of its diverse functions in various human organs, tissues, and cells. In this review, we focused on KLF15 role in kidney biology and highlighted its role as a protective factor against podocyte injury, renal fibrosis, and other pathological processes. As the dysregulation of KLF15 has been shown to be implicated in the progression of CKD, KLF15 is expected to be a novel therapeutic target.

Remarkably, the podocyte-specific induction of Klf15 exhibits a renal-protective role in proteinuric murine models (13). Future studies are warranted to examine whether a prolonged induction is safe and effective, and whether other cell-specific or a global induction will exhibit similar beneficial roles. In 
addition, what roles KLF15 may play in other glomerular or tubular diseases, and which pathway may be involved, also need to be investigated in multiple directions.

Present studies on the role of KLF15 in kidney biology mainly focus on podocyte injury, mesangial pathology, and renal fibrosis. In this review, we have introduced several major functions and the corresponding molecular mechanisms of KLF15, involving the restoration of podocyte differentiation markers, the inhibition of the pathological proliferation of mesangial cells, and the antifibrotic effect in mesangial cells and the tubulointerstitium. Different roles in different cells indicate a cell-specific role of KLF15 in kidneys. The functions and molecular mechanisms of KLF15 in other cells in the kidney need further investigation. Future research concerning the functions of KLF15 in kidney development will promote the understanding of this issue. In addition, since KLF15 regulates target gene expression by interacting with binding partners (25), it would be interesting to know if the function of KLF15 will be lost or changed once these protein-protein interactions are destroyed. Therefore, it will be a significant task to identify the specific protein binding partners and the downstream target of KLF15 in diverse cell types, thus enabling the development of potential therapeutic targets.

\section{Conclusions}

In this review, we summarized the diverse roles of KLF15 and elaborated on its protective effects in kidney diseases (Table 1), providing new insights into the progression and therapy of CKD. KLF15 is expected to be a potential therapeutic target for glomerular diseases and renal fibrosis. Verifying specific functional mechanisms of KLF15 in different cell types or diseases will be a promising prospect in moving forward in precision medicine.

\section{Acknowledgements}

This work was supported by the National key R\&D program of China (2018YFC2000400) and the National Natural Science Foundation of China under grants (81670651, and 31470776).

Table 1. Functions of KLF15 in kidney biology

\begin{tabular}{|c|c|c|c|}
\hline & Experimental model/cell & Functions and relevant mechanisms in kidney biology & Refs \\
\hline $\begin{array}{l}\text { Tubular } \\
\text { physiology }\end{array}$ & Rat tubular cells. & $\begin{array}{l}\text { KLF15 suppressed the expression of CLC-K1 and CLC-K2 in the thin descending limb of the } \\
\text { loop of Henle and inner medullary collecting ducts by competing for promoter binding } \\
\text { with MAZ, contributing to the kidney-specific expression of CLC-K1 and CLC-K2. }\end{array}$ & $(23)$ \\
\hline \multirow[t]{3}{*}{ Podocyte injury } & $\begin{array}{l}\text { Podocyte-specific Klf15-KO mice } \\
\text { treated with/without LPS or ADR; } \\
\text { kidney biopsies from patients with } \\
\text { FSGS and HIVAN. }\end{array}$ & $\begin{array}{l}\text { KLF15 is a key regulator of podocyte differentiation and protects against podocyte injury } \\
\text { via transcriptionally regulating podocyte-specific genes (Synaptopodin, Podocin, and } \\
\text { Nephrin). }\end{array}$ & $(27)$ \\
\hline & $\begin{array}{l}\text { Human podocytes; proteinuric murine } \\
\text { models. }\end{array}$ & $\begin{array}{l}\text { KLF15 is important in mediating the beneficial effects of GCs in podocytes. Loss of KLF15 } \\
\text { led to a destabilization of the actin cytoskeleton and an increased podocyte injury, while } \\
\text { overexpression of KLF15 stabilized the actin cytoskeleton under podocyte stress. }\end{array}$ & $(16)$ \\
\hline & $\begin{array}{l}\text { Podocyte-specific induction of Klf15 in } \\
\text { ADR-induced and HIV-1 transgenic } \\
\text { (Tg26) proteinuric murine models. }\end{array}$ & $\begin{array}{l}\text { KLF15 is renal-protective. Podocyte-specific induction of Klf15 attenuated renal fibrosis, } \\
\text { inflammation and podocyte injury, and improved renal function. Mechanisms include } \\
\text { activation of pathways implicated in the restoration of podocyte differentiation markers, } \\
\text { the stabilization of the actin cytoskeleton, and focal adhesion. }\end{array}$ & $(13)$ \\
\hline $\begin{array}{l}\text { Mesangial } \\
\text { pathology }\end{array}$ & $\begin{array}{l}\text { Classic anti-Thy } 1 \text { mesangial } \\
\text { proliferative nephritis rat model. }\end{array}$ & $\begin{array}{l}\text { KLF15 expression in mesangial cells inhibited cell proliferation by down-regulating the } \\
\text { expression of cell cycle regulation factor E2F1. }\end{array}$ & $(37)$ \\
\hline Lupus Nephritis & In vivo $\mathrm{LN}$ mouse model. & $\begin{array}{l}\text { Combined treatment of Hydroxychloroquine and artemisinin exerted renal-protective } \\
\text { effects by increasing the levels of KLF15, leading to a decreased expression levels of NF- } \mathrm{B} \text {, } \\
\text { and consequently suppressing the inflammatory response. }\end{array}$ & (21) \\
\hline \multirow[t]{5}{*}{ Renal fibrosis } & $\begin{array}{l}\text { UUO mice (progressive renal fibrosis } \\
\text { model); Foxd1-Cre Klf15f/ll cells in UUO } \\
\text { mice and Ang II-treated mice. }\end{array}$ & $\begin{array}{l}\text { KLF15 has an antifibrotic effect in renal fibrosis by inhibiting the canonical Wnt/ } \beta \text {-catenin } \\
\text { pathway. }\end{array}$ & $(46)$ \\
\hline & Ang II-treated mice. & $\begin{array}{l}\text { KLF15 inhibited the expression of Ang II-induced CTGF by suppressing the recruitment of } \\
\text { the co-activator P/CAF to the CTGF promoter, exerting an early antifibrotic effect in renal } \\
\text { fibrosis. }\end{array}$ & $(18)$ \\
\hline & $\begin{array}{l}\text { 5/6-nephrectomized rat; murine } \\
\text { mesangial cells and HEK } 293 \text { cells } \\
\text { transfected with a mouse KLF15 } \\
\text { complementary DNA expression } \\
\text { vector. }\end{array}$ & $\begin{array}{l}\text { The expression of KLF15 in mesangial cells was suppressed in the remnant kidney due to } \\
\text { elevated levels of TGF- } \beta \text {, TNF- } \alpha \text {, and oxidative stress. Overexpression of KLF15 in } \\
\text { mesangial cells and HEK } 293 \text { cells down-regulated the expression of fibronectin and type IV } \\
\text { collagen mRNAs. }\end{array}$ & $(45,47)$ \\
\hline & 5/6-nephrectomized rat. & $\begin{array}{l}\text { KLF15 attenuated the deposition of extracellular matrix and CTGF by inhibiting } \\
\text { TGF- } \beta 1 \text {-mediated JNK/MAPK and ERK/MAPK signaling pathways. Thus, KLF15 plays an } \\
\text { antifibrotic role in renal interstitial fibrosis. }\end{array}$ & (17) \\
\hline & MPC- 5 cells. & $\begin{array}{l}\text { KLF15 protects against hypertensive nephropathy by decreasing the expression levels of } \\
\text { fibronectin and type IV collagen. }\end{array}$ & $(48)$ \\
\hline
\end{tabular}




\section{Abbreviations}

KLF15: Krüppel-like factor 15; KO: knockout; LPS: lipopolysaccharide; ADR: Adriamycin; FSGS: Focal Segmental Glomerulosclerosis; HIVAN: HIV-associated nephropathy; GCs: Glucocorticoids;

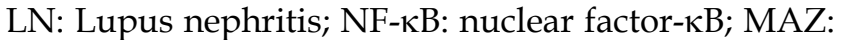
myc-associated zinc-finger; UUO: unilateral ureteric obstruction; Ang II: angiotensin II; CTGF: connective tissue growth factor; TGF- $\beta$ : transforming growth factor- $\beta$; TNF- $\alpha$ : tumor necrosis factor- $\alpha$; ERK: extracellular-regulated kinase; MAPK: mitogen-activated protein kinase; JNK: Jun N-terminal kinase; MPC-5 cells: conditionally immortalized mouse podocytes; P/CAF: p300/CREB-binding protein-associated factor; RA: retinoic acid; cAMP: cyclic adenosine monophosphate; PKA: protein kinase A; CRE: cAMP response element; CREB: cAMP response element-binding protein; WT1: Wilms Tumor 1; GR: glucocorticoids receptor; GRE: GC response element; CDK2: cyclin-dependent kinase 2.

\section{Competing Interests}

The authors have declared that no competing interest exists.

\section{References}

1. Murphy D, McCulloch CE, Lin F, Banerjee T, Bragg-Gresham JL, Eberhardt MS, et al. Trends in Prevalence of Chronic Kidney Disease in the United States. Annals of internal medicine. 2016; 165: 473-81.

2. Glassock RJ, Warnock DG, Delanaye P. The global burden of chronic kidney disease: estimates, variability and pitfalls. Nature reviews Nephrology. 2017; 13: 104-14.

3. Bruck K, Stel VS, Gambaro G, Hallan S, Volzke H, Arnlov J, et al. CKD Prevalence Varies across the European General Population. J Am Soc Nephrol. 2016; 27: 2135-47.

4. Zhang L, Wang F, Wang L, Wang W, Liu B, Liu J, et al. Prevalence of chronic kidney disease in China: a cross-sectional survey. Lancet (London, England). 2012; 379: 815-22.

5. Drawz P, Rahman M. Chronic kidney disease. Annals of internal medicine. 2015; 162: Itc1-16.

6. Stel VS, Bruck K, Fraser S, Zoccali C, Massy ZA, Jager KJ. International differences in chronic kidney disease prevalence: a key public health and epidemiologic research issue. Nephrol Dial Transplant. 2017; 32: 129-35.

7. Humphreys BD. Mechanisms of Renal Fibrosis. In: Julius D, editor. Annual Review of Physiology, Vol 80. Palo Alto: Annual Reviews; 2018. p. 309-26.

8. Peralta CA, Lin F, Shlipak MG, Siscovick D, Lewis C, Jacobs DR, et al. Race differences in prevalence of chronic kidney disease among young adults using creatinine-based glomerular filtration rate-estimating equations. Nephrol Dial Transplant. 2010; 25: 3934-9.

9. Ecder T. Early diagnosis saves lives: focus on patients with chronic kidney disease. Kidney Int Suppl. 2013; 3: 335-6.

10. Nelson, Tuttle, Bilous. KDOQI Clinical Practice Guideline for Diabetes and CKD: 2012 Update (vol 60, pg 850, 2012). Am J Kidney Dis. 2013; 61: 1049.

11. Araujo M, Doi SQ. Editorial: Biomarkers in CKD. Front Med. 2017; 4: 2

12. Mallipattu SK, Estrada CC, He JC. The critical role of Krüppel-like factors in kidney disease. American journal of physiology Renal physiology. 2017; 312: F259-f65.

13. Guo Y, Pace J, Li Z, Ma'ayan A, Wang Z, Revelo MP, et al. Podocyte-Specific Induction of Krüppel-Like Factor 15 Restores Differentiation Markers and Attenuates Kidney Injury in Proteinuric Kidney Disease. Journal of the American Society of Nephrology: JASN. 2018; 29: 2529-45.

14. Kim CK, He P, Bialkowska AB, Yang VW. SP and KLF Transcription Factors in Digestive Physiology and Diseases. Gastroenterology. 2017; 152: 1845-75.

15. Fan $\mathrm{Y}, \mathrm{Lu} \mathrm{H}$, Liang $\mathrm{W}, \mathrm{Hu} \mathrm{W}$, Zhang J, Chen YE. Krüppel-like factors and vascular wall homeostasis. Journal of molecular cell biology. 2017; 9: 352-63.

16. Mallipattu SK, Guo Y, Revelo MP, Roa-Pena L, Miller T, Ling J, et al. Krüppel-Like Factor 15 Mediates Glucocorticoid-Induced Restoration of
Podocyte Differentiation Markers. Journal of the American Society of Nephrology: JASN. 2017; 28: 166-84

17. Gao X, Wu G, Gu X, Fu L, Mei C. Krüppel-like factor 15 modulates renal interstitial fibrosis by ERK/MAPK and JNK/MAPK pathways regulation. Kidney \& blood pressure research. 2013; 37: 631-40.

18. Gu X, Xu D, Fu L, Wang Y, Mei C, Gao X. KLF 15 Works as an Early Anti-Fibrotic Transcriptional Regulator in Ang II-Induced Renal Fibrosis via Down-Regulation of CTGF Expression. Kidney \& blood pressure research. 2017; 42: 999-1012.

19. Takeuchi Y, Yahagi N, Aita Y, Murayama Y, Sawada Y, Piao X, et al. KLF15 Enables Rapid Switching between Lipogenesis and Gluconeogenesis during Fasting. Cell reports. 2016; 16: 2373-86.

20. Matoba K, Lu Y, Zhang R, Chen ER, Sangwung P, Wang B, et al. Adipose KLF15 Controls Lipid Handling to Adapt to Nutrient Availability. Cell reports. 2017; 21: 3129-40

21. Liang N, Zhong Y, Zhou J, Liu B, Lu R, Guan Y, et al. Immunosuppressive effects of hydroxychloroquine and artemisinin combination therapy via the nuclear factor-kappaB signaling pathway in lupus nephritis mice. Experimental and therapeutic medicine. 2018; 15: 2436-42.

22. Uchida S, Tanaka Y, Ito H, Saitoh-Ohara F, Inazawa J, Yokoyama KK, et al. Transcriptional regulation of the CLC-K1 promoter by myc-associated zinc finger protein and kidney-enriched Krüppel-like factor, a novel zinc finger repressor. Molecular and cellular biology. 2000; 20: 7319-31.

23. Uchida S, Sasaki S, Marumo F. Isolation of a novel zinc finger repressor that regulates the kidney-specific CLC-K1 promoter. Kidney international. 2001; 60: 416-21

24. Kim YD, Hwang SL, Jeon HJ, Jeon YH, Nedumaran B, Kim K, et al. B-cell translocation gene 2 enhances fibroblast growth factor 21 production by inducing Kruppel-like factor 15. Scientific reports. 2019; 9: 3730.

25. Rane MJ, Zhao Y, Cai L. Krüppel-like factors (KLFs) in renal physiology and disease. EBioMedicine. 2019.

26. Srivastava T, Sharma M, Yew KH, Sharma R, Duncan RS, Saleem MA, et al. LPS and PAN-induced podocyte injury in an in vitro model of minimal change disease: Changes in TLR profile. J Cell Commun Signal. 2013; 7: 49-60.

27. Mallipattu SK, Liu R, Zheng F, Narla G, Ma'ayan A, Dikman S, et al. Krüppel-like factor 15 (KLF15) is a key regulator of podocyte differentiation. The Journal of biological chemistry. 2012; 287: 19122-35.

28. Yang SH, Choi JW, Huh D, Jo HA, Kim S, Lim CS, et al. Roles of fluid shear stress and retinoic acid in the differentiation of primary cultured human podocytes. Experimental cell research. 2017; 354: 48-56.

29. Ivy JR, Drechsler M, Catterson JH, Bodmer R, Ocorr K, Paululat A, et al. 29Nephrocytes. PloS one. 2015; 10: e0134620.

30. Weavers H, Prieto-Sanchez S, Grawe F, Garcia-Lopez A, Artero R, Wilsch-Brauninger $\mathrm{M}$, et al. The insect nephrocyte is a podocyte-like cell with a filtration slit diaphragm. Nature. 2009; 457: 322-6.

31. Barton M, Sorokin A. Endothelin and the glomerulus in chronic kidney disease. Seminars in nephrology. 2015; 35: 156-67.

32. Meyrier A. Mechanisms of disease: focal segmental glomerulosclerosis. Nature clinical practice Nephrology. 2005; 1: 44-54.

33. Nagata M. Podocyte injury and its consequences. Kidney international. 2016; 89: 1221-30.

34. Han SS, Yu MY, Yoo KD, Lee JP, Kim DK, Kim YS, et al. Loss of KLF15 accelerates chronic podocyte injury. International Journal of Molecular Medicine. 2018; 42: 1593-602.

35. van Husen $M$, Kemper MJ. New therapies in steroid-sensitive and steroid-resistant idiopathic nephrotic syndrome. Pediatric nephrology (Berlin, Germany). 2011; 26: 881-92.

36. Zhang J, Zhong HB, Lin Y, Yao W, Huang JY. KLF15 suppresses cell proliferation and extracellular matrix expression in mesangial cells under high glucose. International journal of clinical and experimental medicine. 2015; 8: 20330-6.

37. Hong $\mathrm{O}, \mathrm{LiC}$, Xie $\mathrm{Y}, \mathrm{Lv}$ Y, Liu X, Shi S, et al. Krüppel-like factor-15 inhibits the proliferation of mesangial cells. Cellular physiology and biochemistry: international journal of experimental cellular physiology, biochemistry, and pharmacology. 2012; 29: 893-904.

38. Arias LF, Taborda-Murillo A. Mesangial proliferative glomerulonephritis: A glomerular disease or a non-specific morphological change? Nephrology (Carlton, Vic). 2017; 22: 575.

39. Almaani S, Meara A, Rovin BH. Update on Lupus Nephritis. Clinical journal of the American Society of Nephrology: CJASN. 2017; 12: 825-35.

40. Anders HJ, Rovin B. A pathophysiology-based approach to the diagnosis and treatment of lupus nephritis. Kidney international. 2016; 90: 493-501.

41. Parikh SV, Rovin BH. Current and Emerging Therapies for Lupus Nephritis. Journal of the American Society of Nephrology: JASN. 2016; 27: 2929-39.

42. Lu Y, Zhang L, Liao X, Sangwung P, Prosdocimo DA, Zhou G, et al. Krüppel-like factor 15 is critical for vascular inflammation. J Clini Invest. 2013; 123: 4232-41

43. Edeling M, Ragi G, Huang S, Pavenstadt H, Susztak K. Developmental signalling pathways in renal fibrosis: the roles of Notch, Wnt and Hedgehog. Nature reviews Nephrology. 2016; 12: 426-39.

44. Liu Y. Cellular and molecular mechanisms of renal fibrosis. Nature reviews Nephrology. 2011; 7: 684-96.

45. Gao X, Huang L, Grosjean F, Esposito V, Wu J, Fu L, et al. Low-protein diet supplemented with ketoacids reduces the severity of renal disease in 5/6 nephrectomized rats: a role for KLF15. Kidney international. 2011; 79: 987-96. 
46. Gu X, Mallipattu SK, Guo Y, Revelo MP, Pace J, Miller T, et al. The loss of Krüppel-like factor 15 in Foxd1(+) stromal cells exacerbates kidney fibrosis. Kidney international. 2017; 92: 1178-93.

47. Wang Y, Mitch WE. Proteins and renal fibrosis: low-protein diets induce Krüppel-like factor-15, limiting renal fibrosis. Kidney international. 2011; 79: 933-4.

48. Li N, Zhang J, Yan X, Zhang C, Liu H, Shan X, et al. SIRT3-KLF15 signaling ameliorates kidney injury induced by hypertension. Oncotarget. 2017; 8: 39592-604. 